\author{
Dissertação apresentada à \\ Escola Politécnica da \\ Universidade de São Paulo para \\ obtenção de título de Mestre em \\ Engenharia
}

São Paulo

2012 


\section{REDUÇÃO DOS IMPACTOS AMBIENTAIS CAUSADOS POR EMISSÕES DE GASES NO TRANSPORTE MARÍTIMO}

Dissertação apresentada à

Escola Politécnica da

Universidade de São Paulo para

obtenção de título de Mestre em

Engenharia

Área de Concentração:

Engenharia Naval e Oceânica

Orientador:

Professor Dr.

Hernani Brinati

São Paulo

2012 
Este exemplar foi revisado e alterado em relação à versão original, sob responsabilidade única do autor e com a anuência de seu orientador.

São Paulo, 07 de janeiro de 2012.

Assinatura do autor

Assinatura do orientador

FICHA CATALOGRÁFICA

Montoya Cisneros, Juan Carlos

Redução dos impactos ambientais causados por emissões de gases no transporte marítimo / J.C. Montoya Cisneros. ed.rev. -- São Paulo, 2012.

$103 \mathrm{p}$.

Dissertação (Mestrado) - Escola Politécnica da Universidade de São Paulo. Departamento de Engenharia Naval e Oceânica.

1.Transporte marítimo 2.Impactos ambientais 3.Gases (Emissão) I.Universidade de São Paulo. Escola Politécnica. Departamento de Engenharia Naval e Oceânica II.t. 


\section{DEDICATÓRIA}

A minha esposa, Paola Sofía, sem ela nenhum sonho seria possível ou valeria a pena realizar. A meus filhos Luciana e Thiago, luzes da minha vida. 


\section{AGRADECIMENTOS}

Agradeço principalmente a Deus.

Agradeço em forma muito especial ao Prof. Dr. Hernani Luiz Brinati, pela orientação, por ter transmitido seu conhecimento, compartilhado seu tempo, ter confiado na minha aprendizagem e em especial pela amizade.

A minha esposa Paola pelo apoio incondicional; não esqueça, que suceda o que suceder eu vou amar você toda minha vida, já que você é minha outra metade. Eu amo você mais que ontem e menos que amanhã.

Ao Prof. Dr. Moyses Szajnbok, pela sua sabedoria emanada nas nossas conversas, sua bondade de servir, orientar e transmitir sua experiência.

Ao Departamento de Engenharia Naval e Oceânica da Escola Politécnica da Universidade de São Paulo em especial a Lânia Camilo pelo apoio e grata disposição.

A minha mãe Libia Isabel e meu pai Manuel Ricardo por fazer de mim um homem com princípios.

A Vanesa por acudir a nosso chamado quando precisamos dela. 


\section{RESUMO}

O transporte marítimo depende essencialmente da energia dos derivados do petróleo e, em conseqüência, produz emissões de $\mathrm{CO}_{2}, \mathrm{SO}_{x}, \mathrm{NO}_{\mathrm{x}}$ e material particulado entre outros. Hoje 0 transporte aquaviário responde por aproximadamente $4,5 \%$ das emissões de carbono, $4 \%$ das emissões de óxidos de enxofre e $7 \%$ das emissões de oxido de nitrogênio. Se a expansão desse modal de transporte se mantiver nos próximos anos, como se tem previsto, a sua participação na emissão destes tipos de poluentes deve aumentar e, certamente, haverá pressões da sociedade para introdução de medidas que contenham esse aumento.

Pode-se afirmar que os poluentes gerados pelo Transporte Marítimo são resultado de deficiência em projeto de navios e de seus sistemas, de uma má gestão operacional, e inclusive da ausência de medidas de regulamentação mais rigorosas. $\mathrm{O}$ estudo se concentra em estudar dois destes pontos.

O primeiro se refere ao desenvolvimento do projeto do navio e da instalação propulsora com a preocupação de reduzir o consumo de energia. Isso implica em alterações nos projetos e seus sistemas de propulsão, tirando melhor proveito da tecnologia disponível no mercado, bem como análise da viabilidade de uso de fontes alternativas de energia. O segundo ponto se refere ao processo de gestão operacional, com ênfase na redução da emissão de poluentes. Serão examinados exemplos práticos que sustentem as reduções e benefícios, por exemplo, a redução de velocidade do navio.

Por ultimo foram expostas as alternativas que o autor considera as mais promissoras para atingir o objetivo pretendido. São selecionadas as melhores opções que contribuem para a redução das emissões de gases, entre as medidas tecnológicas, operacionais e de dimensionamento de frota. Algumas destas alternativas podem ser aplicadas aos navios existentes, enquanto que outras só podem ser aplicadas no projeto de navios novos. 


\section{ABSTRACT}

The shipping depends on the energy of oil products, which produce emissions of $\mathrm{CO}_{2}, \mathrm{SO}_{\mathrm{x}}, \mathrm{NO}_{\mathrm{x}}$ and particulate matter. Today, the water transport accounts for approximately $4.5 \%$ of carbon emissions, $4 \%$ of sulfur oxides emissions and $7 \%$ of nitrogen oxide emissions. If the expansion of this mode of transport is maintained in the coming years, as has been predicted, its participation in the emission of these types of pollutants must increase, and certainly there will be pressure from society for introducing measures to contain its increase. It can be argued that pollutants generated by the Maritime Transport are the result of deficiency in the design of ships and theirs systems, deficient operational management, and even the absence of more stringent regulatory measures. The study focuses on the study of these two points.

The first refers to the development of Ship design and propulsion plant with a view to reducing energy consumption. This implies changes in design and propulsion systems, making best use of available technology, as well as the evaluation of viability in the use of sources of alternative energy. The second point refers to the process of operational management, with emphasis on reducing of emissions. Practical examples are examined to support and benefit the reductions of emissions, for example, the reduction of ship speed.

Finally is exposed the alternatives that the author considers the most promising to achieve the desired goal. The best options are selected to reducing greenhouse gas emissions between technological measures, operational and fleet sizing. Some of these alternatives can be applied to existing ships, while others may only be applied in the design of new ships. 


\section{SUMÁRIO}

CAPÍTULO 1: INTRODUÇÃO........................................................................

1.1 Definição do problema....................................................................

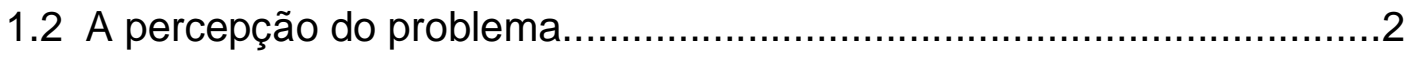

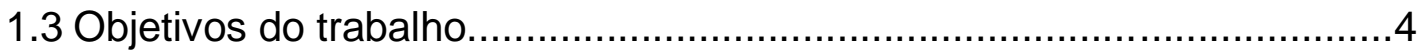

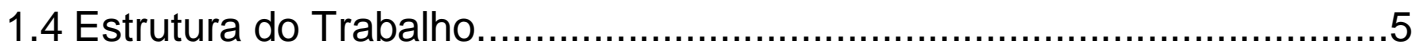

\section{CAPÍTULO 2: 0 TRANSPORTE MARÍTIMO E SEUS IMPACTOS}

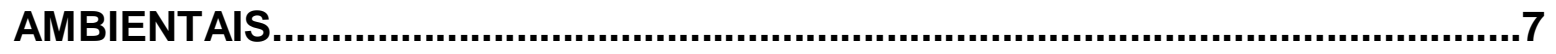

2.1. Analise do transporte Marítimo na Atualidade .....................................

2.2. Tipos de impactos gerados pelo transporte marítimo ...........................11

2.2.1. Águas de lastro...........................................................12

2.2.2. Hidrocarbonetos e Águas Oleosas......................................12

2.2.3. Águas Residuais.........................................................13

2.2.4. Águas Cinzas....................................................................13

2.2.5. Resíduos Sólidos ou Lixo Marinho........................................14

2.2.6. Pinturas Navios............................................................... 14

2.3. Avaliação dos impactos das emissões de gases dos motores..............15

2.3.1. Considerações Preliminares................................................15

2.3.2 Combustíveis Usados na Propulsão Naval.............................16 
2.3.3. Tipos de Emissões de Gases Geradas pelos Navios .23

2.3.4. Efeitos causados pelas emissões na saúde humana e no clima mundial.

CAPÍTULO 3: MARCO NORMATIVO INTERNACIONAL .31

3.1. Atuação da Organização Marítima Internacional. 31

3.2. Convênio Internacional para a Prevenção da Contaminação pelos Navios (MARPOL73/78) 32

3.3. Anexo VI da MARPOL $73 / 78$ Regras para a Prevenção da Poluição do Ar Causada por Navios. .34

3.3.1 Considerações Gerais sobre as Normas 34

3.3.2 Analise do Anexo VI do MARPOL "Regras para a Prevenção da Poluição do Ar Causada por Navios"..... .36

3.3.3 O Anexo VI e o CO2 (Principal gás de efeito estufa) 39

3.3.4. Comparação com os limites de emissões dos caminhões da União Européia. 39

CAPÍTULO 4: MEDIDAS PARA REDUÇÃO DE EMISSÕES .42

4.1. Medidas Tecnológicas 42

4.1.1 Primeira linha: Alternativas para a redução da potencia requerida .43

4.1.2 Segunda linha: Modificações no projeto do motor. .44

4.1.3 Terceira linha: Uso de outras fontes de energia .57 
4.2. Adoção de Medidas Operacionais..................................................68

4.2.1 Programa de manutenção..................................................68

4.2.2 Planejamento de viagens.................................................

4.3. Revisão dos conceitos de dimensionamento da frota.........................74

\section{CAPÍTULO 5: AVALIAÇÃO DAS ALTERNATIVAS PARA REDUÇÃO DAS

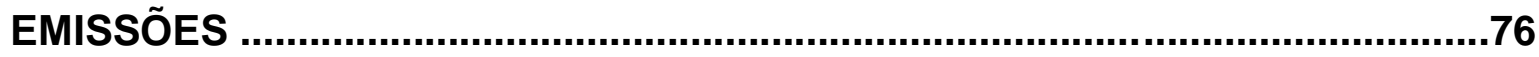

5.1. Considerações Preliminares........................................................

5.2. Avaliação das medidas tecnológicas.............................................79

5.3. Avaliação das medidas operacionais.............................................. 82

5.4, Dimensionamento da frota.......................................................... 83

5.4.1 Redução de velocidade: um exemplo ilustrativo....................83

CAPÍTULO 6: CONCLUSÕES E RECOMENDAÇÕES .....................................94

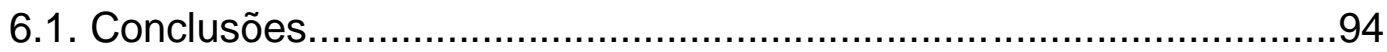

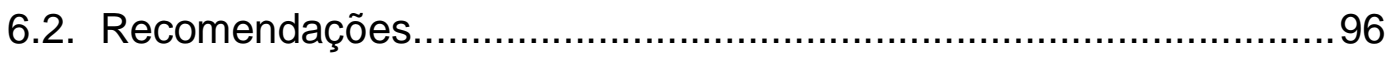

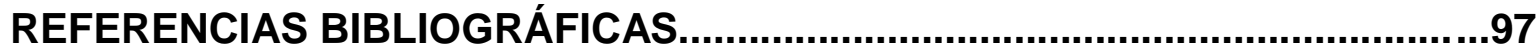




\section{LISTA DE ABREVIATURAS E SÍMBOLOS}

UNCTAD United Nations Conference on Trade and Development

OMI Organização Marítima Internacional

MARPOL Maritime Pollution

MEPC Comitê de Proteção do Meio Marinho

UE União Européia

OMC Organização Mundial do Comercio

OMS Organização Mundial da Saúde

OCIMF Marítimo Internacional de Companhias Petroleiras

M.G.O. Marine Gás Oil

M.D.O. Marine Diesel Oil

L.M.F.O. Light Marine Fuel oil

M.F.O. Marine Fuel oil

LNG Liquefied natural Gás ou Gás Natural Liquefeito

$\mathrm{MCl} \quad$ Motor de Combustão Interna

ISO International Organization for Standardization

O Oxigênio

$\mathrm{N} \quad$ Nitrogênio

CO Monóxido de Carbono

$\mathrm{CO}_{2} \quad$ Dióxido de Carbono

$\mathrm{SO}_{\mathrm{x}} \quad$ Óxidos de Enxofre

$\mathrm{SO}_{2} \quad$ Dióxido de Enxofre

$\mathrm{NO}_{\mathrm{x}} \quad$ Óxidos de Nitrogênio

MP Material Particulado

$\mathrm{HC} \quad$ Hidrocarbonetos

$\mathrm{PCl} \quad$ Poder Calorífico Inferior 


\section{LISTA DE FIGURAS}

Figura 2.1 Taxa de variação de uso entre vela, carvão e petróleo..........................16

Figura 2.2 Entradas e saídas de um motor de combustão interna........................24

Figura 2.3 Entradas e saídas de um motor diesel de baixa rotação.......................26

Figura 2.4 Emissões de gases de descarga típicas de um motor diesel de media

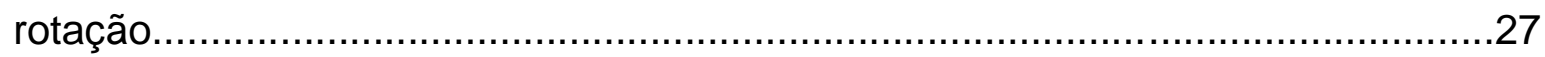

Figura 2.5 Densidade do Trefego do Transporte Marítimo Mundial.......................30

Figura 3.1 Evolução das restrições à emissão de NOx.........................................36

Figura 4.1 Sistema de Motor de Ar Úmido (HAM) MAN B\&W..............................46

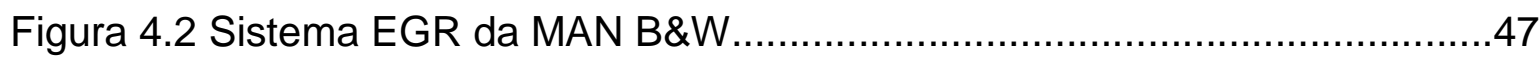

Figura 4.3 Sistema de Injeção de Água DWI (Wärtsilä)......................................49

Figura 4.4 Simulação em CFD com Injeção Direta de Água (DWI) e sem ela.......50

Figura 4.5 Processo da Redução Catalítica Seletiva (SCR). MAN B\&W..............52

Figura 4.6 Esquema SCR, planta integrada com sistema de Turbo- alimentação da

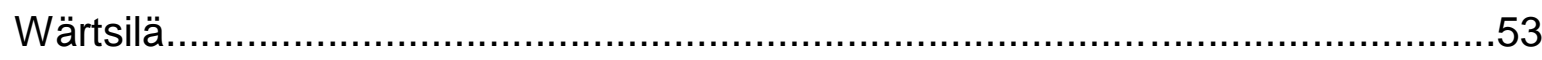

Figura 4.7 Conceição da THERMO EFFICIENCY SYSTEMS (TES) desenvolvida

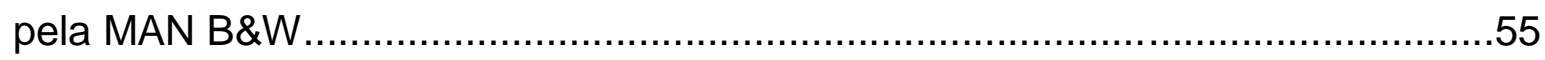

Figura 4.8 Conceição da WASTE HEAT RECOVERY (WHR) desenvolvida pela

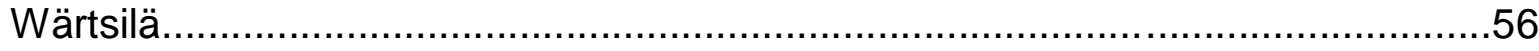

Figura 4.9 Diversas plataformas para aproveitar a energia eólica.......................61

Figura 4.10 Plataforma tipo pipa aproveitada pela companhia SkySails..............62 
Figura 4.11 Planta a propulsão elétrica dual da Wärtsilä. .64

Figura 4.12 Comparação dos poluentes $\left(\mathrm{SO}_{\mathrm{x}}, \mathrm{CO}_{2}, \mathrm{NO}_{\mathrm{x}}\right)$ com diferentes tipos de instalação. 64

Figura 4.13 Reação da pilha de combustível para formar eletricidade. 66

Figura 4.14 Painéis Solares no cargueiro NYK Auriga Leader, eleito o "Navio do Ano" pela Premiação Global 2009 da Lloyd's List..... .67

Figura 4.15 Velocidade do navio em função da Rugosidade do Casco. 69

Figura 4.16 Incremento de Potência/Combustível em função da Rugosidade do Casco....... .70

Figura 4.17 Comparação dos custos operativos de um navio Panamax e um post-

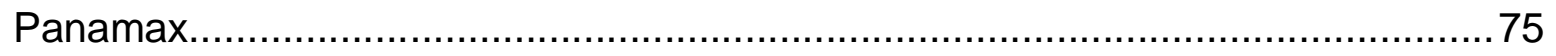

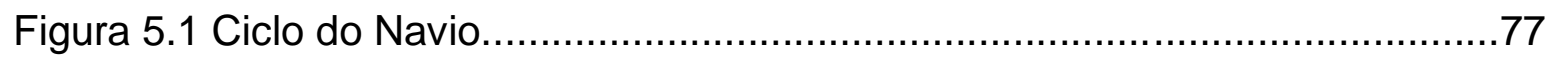

Figura 5.2 Focos do Projeto do Navio............................................................. 


\section{LISTA DE TABELAS}

Tabela 2.1 Crescimento em volume do Comercio de Mercadorias por regiões 2005-2007.

Tabela 2.2 Evolução do Tráfico Marítimo Internacional (Mercadorias Carregadas) Milhões de Toneladas.

Tabela 2.3 Frota Mundial em milhões de toneladas deadweight (porcentagem)...10

Tabela 2.4 Nomes mais comuns dos Combustíveis marinhos.

Tabela 2.5 Propriedade física dos Combustíveis (Study European Commission2002). 19

Tabela 2.6 ISO 8217: Especificações técnicas para combustíveis destilados tipo DMX, DMA, DMZ e DMB .20

Tabela 2.7 ISO 8217: Especificações técnicas para combustíveis residuais de tipo RMA, RMB, RMD e RME .21

Tabela 2.8 ISO 8217: Especificações técnicas para combustíveis residuais de tipo RMG e RMK. 22

Tabela 3.1 Comparação de Limites de emissões para $\mathrm{NO}_{x}$ e limite de conteúdo de enxofre de Caminhões (EURO) e Navios (OMI) gr/Kw.hr para NOx e ppm para S. .40

Tabela 4.1 Redução do consumo de combustível mediante otimização do caso..43

Tabela 4.2 Composição do LNG de acordo com sua origem. .57

Tabela 4.3 Comparação de $\mathrm{SO}_{x}, \mathrm{CO}_{2}, \mathrm{NO}_{x}$ dos diferentes tipos de instalação....65

Tabela 5.1 Benefícios da otimização do projeto do navio. 79 
Tabela 5.2 Quadro comparativo dos resultados obtidos...................................89

Tabela 5.3 Quantidade de Emissões de um Motor de Baixa Rotação (g/Kwh).. 90

Tabela 5.4 Emissão de poluentes geradas pelo navios $\mathrm{N} 1$..................................90

Tabela 5.5 Emissão de poluentes geradas pelo navios N2 ................................91

Tabela 5.6 Emissão de poluentes geradas em fila e portos para os navios...........91

Tabela 5.7 Comparação das Emissões para os Navios N1 e N2 ..........................92

Tabela 5.8 Comparação das Emissões entre as Frota 1 e 2.................................93 